\title{
MODELAGEM E INVESTIGAÇÃO DE PERIODICIDADES NAS TAXAS DE INTERNAÇÕES POR DIARREIA ANTES E APÓS A VACINA CONTRA O ROTAVÍRUS NO ESTADO DO PARANÁ
}

\author{
Bruno Felipe de Jesus Oliveira Crislaine Menezes da Silva* \\ Rafael Gutierres Castanha Gabriela de Oliveira Nascimento Brassarote ${ }^{\dagger}$ \\ Pós Graduação em Matemática Aplicada e Computacional, FCT/UNESP \\ 19060-900, Presidente Prudente, SP \\ E-mail: b-fellipe@hotmail.com, crismenezes_@live.com, r.castanha@gmail.com, gabriela.2308@hotmail.com \\ Eniuce Menezes de Souza Taqueco T. Uchimura Maria de L. T. Masukawa \\ Departamento de Estatística, Pós graduação em Bioestatística,UEM \\ 87020-900, Maringá, PR \\ E-mail: emsouza@uem.br, taqueco@gmail.com, mltmasukawa@hotmail.com
}

\begin{abstract}
$\underline{\text { RESUMO }}$
No estudo de séries temporais no domínio de frequências, a análise de Fourier ou análise harmônica é bastante utilizada para encontrar periodicidades desconhecidas. Tal procedimento decompõe a série em componentes periódicos, ou seja, a análise de Fourier corresponde à partição da variabilidade da série em componentes de frequências.

Logo, como por ser visto em [2], considerando o modelo da série temporal dado por uma combinação linear de harmônicos (componentes senoidais) da seguinte forma

$$
Z_{t}=\frac{A_{0}}{2} \sum_{j=1}^{m} A_{j} \cos \left(2 \pi f_{j} t\right)+B_{j} \sin \left(2 \pi f_{j} t\right)
$$

onde as frequências $0<f_{1}<f_{2}<\ldots<f_{m}<\frac{1}{2}$ são fixadas e, $A_{j}$ e $B_{j}$ são variáveis aleatórias normais independentes com média zero e $\operatorname{Var}\left(A_{j}\right)=\operatorname{Var}\left(B_{j}\right)=\sigma_{j}^{2}$, sendo calculadas via transformada de Fourier discreta da série. Como todas as frequências de Fourier estão indicadas no modelo acima, o termo aleatório do modelo foi omitido, embora esteja presente quando apenas os harmônicos que realmente contribuem com a série são mantidos.

Nesse sentido, o presente trabalho consiste em realizar uma modelagem harmônica em busca de periodicidades no comportamento temporal das taxas de hospitalização (THs) por diarreia aguda em crianças menores de um ano nas seis macrorregionais do estado do Paraná: Leste, Campos Gerais, Centro Sul, Oeste, Noroeste e Norte. Os dados foram retirados do DATASUS (Departamento de Informática do Sistema Único de Saúde) e o período considerado foi de 2000 a 2011.

Para validação dos resultados, foi utilizado o teste de Fisher (teste para periodicidades) [3]. Tal teste apresenta um procedimento para analisar as periodicidades da série, utilizando as ordenadas do periodograma, sendo esta uma função que estabelece a forma como a variabilidade total da série é particionada ao longo das várias componentes relativas a cada uma das frequências de Fourier.
\end{abstract}

\footnotetext{
*bolsista de mestrado FAPESP

${ }^{\dagger}$ bolsista de mestrado FAPESP
} 
Na Figura 1 são apresentadas as séries temporais (em cinza), bem como os modelos harmônicos ajustados (em preto). Foram considerados apenas os 5 primeiros harmônicos. Os demais foram desconsiderados por não serem estatisticamente significativos. Uma vez que sazonalidades determinísticas foram consideradas e a modelagem harmônica não é localizada temporalmente, a modelagem deve ser realizada em duas etapas, antes (2000 a 2005) e após (2007 a 2011) a introdução na vacina. Devido à vacinação ter iniciado em 2006, este é um ano de transição e, portanto, não foi considerado nessa modelagem.

A partir da modelagem harmônica, verificou-se que antes da vacinação a região Leste tinha um comportamento diferenciado das demais no estado, apresentando uma periodicidade de 12 meses, cujo harmônico foi responsável por $89.06 \%$ da variabilidade da série. O comportamento diferenciado da região Leste, a qual inclui os municípios de Curitiba e Paranaguá, possui condições climáticas específicas que favorecem os picos nos meses de junho. Em relação as demais macrorregiões, o harmônico relativo a periodicidade de 6 meses foi responsável por pelo menos $63 \%$ da variabilidade da série, chegando a atingir $78 \%$. A partir da modelagem foram identificados dois picos no ano, um dominante em agosto e o segundo em fevereiro.

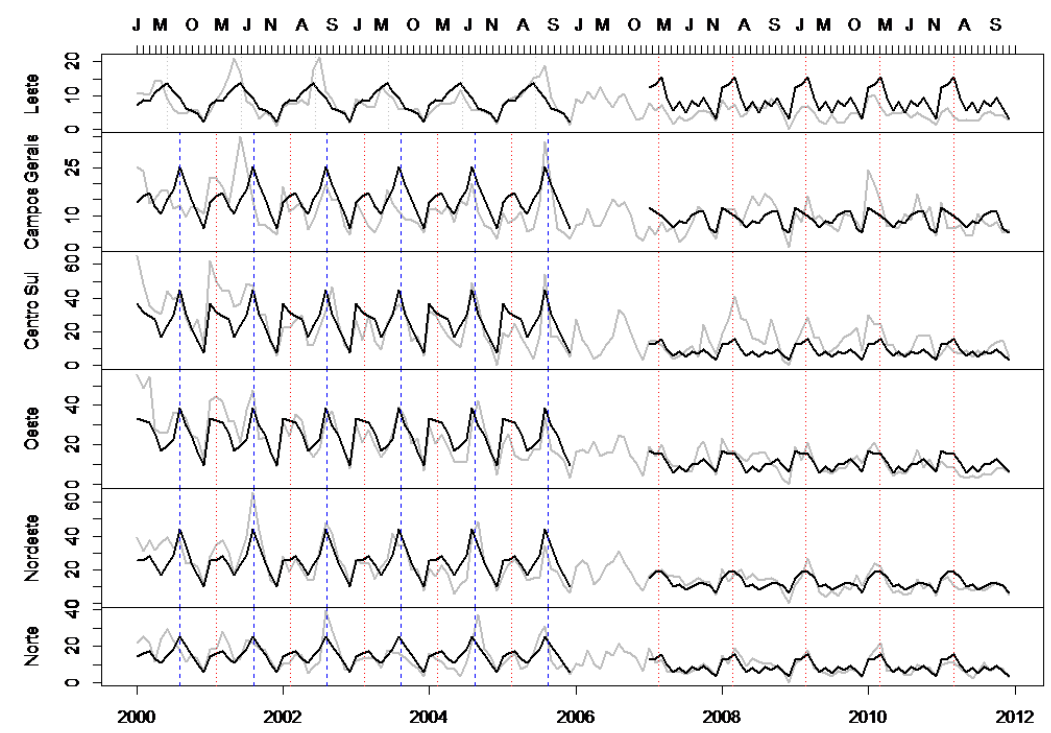

Figura 1: Modelagem harmônica realizada nos dados obtidos por taxas de hospitalização (THs) causadas por diarreia aguda antes a após a vacinação nas 6 macrorregiões do estado do Paraná.

Pelo teste de Fisher confirmou-se que a periodicidade de 12 meses foi estatisticamente significativa $(p<0.01)$ para região Leste, e o mesmo verificou-se com a periodicidade de 6 meses nas demais macrorregiões antes da vacinação.

Após o início da vacinação, todas as macrorregiões apresentaram uma periodicidade de 6 meses. Embora tenha ficado menos evidente, inclusive não estatisticamente significativo $(p>0.05)$, a maioria das macrorregiões passou a apresentar, em geral, maior frequência nos meses de fevereiro. Verificou-se, portanto, uma modificação nas periodicidades das internações, o que era esperado no caso de eficácia da vacina, já que o rotavirus realmente apresenta um comportamento sazonal.

Palavras-chave: Séries Temporais, Análise de Fourier, Teste de Fisher.

\section{Referências}

[1] C. CHATFIELD, "The Analysis of Times Series: An Introduction”, Chapman and Hall. 2003.

[2] J. D. CRYER; K. S. CHAN,“Time Series Analysis With Application in R”, Springer, 2008

[3] P. A. MORETIN; C. M. C. TOLOI, “Análise de Séries Temporais”, Edgard Blücher, 2004. 